\title{
Charles Baudelaire, I fiori del male
}

\section{Mario Richter}

\section{(2) OpenEdition}

\section{Journals}

\section{Edizione digitale}

URL: http://journals.openedition.org/studifrancesi/8025

DOI: 10.4000/studifrancesi.8025

ISSN: 2427-5856

\section{Editore}

Rosenberg \& Sellier

\section{Edizione cartacea}

Data di pubblicazione: 1 juillet 2009

Paginazione: 429

ISSN: 0039-2944

\section{Notizia bibliografica digitale}

Mario Richter, «Charles Baudelaire, I fiori del male», Studi Francesi [Online], 158 (LIII | II) | 2009, online dal 30 novembre 2015, consultato il 09 janvier 2021. URL: http://journals.openedition.org/studifrancesi/ 8025 ; DOI: https://doi.org/10.4000/studifrancesi.8025

Questo documento è stato generato automaticamente il 9 janvier 2021.

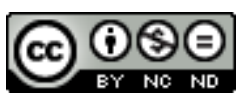

Studi Francesi è distribuita con Licenza Creative Commons Attribuzione - Non commerciale - Non opere derivate 4.0 Internazionale. 


\title{
Charles Baudelaire, I fiori del male
}

\author{
Mario Richter
}

\section{NOTIZIA}

CHARLES BAUDELAIRE, I fiori del male, traduzione di Giorgio CAPRONI, introduzione e commento di Luca PIETROMARCHI, con testo a fronte, testo della traduzione stabilito da Luciano CARCERERI, Venezia, Marsilio, 2008, pp. 567.

1 Potrà non sorprendere nessuno una nuova traduzione italiana delle Fleurs du mal, l'opera poetica francese che certamente annovera il più alto numero di traduttori. In realtà, questa volta ci troviamo di fronte a un evento letterario di sicuro rilievo, perché la traduzione è dovuta a uno dei massimi poeti del nostro Novecento, Giorgio Caproni. Se qualcuno avesse ancora ricordo della traduzione pubblicata dall'editore Curcio sotto il nome di Caproni nell'ormai lontano 1962, occorre subito avvertirlo che il testo di traduzione qui egregiamente proposto è un inedito, tanto più interessante in quanto Caproni rifiutò il testo sul quale il primo editore intervenne arbitrariamente contro le intenzioni dell'autore. È pienamente condivisibile la rinuncia ad una traduzione in versi, che riprende la medesima opzione che conferisce valore anche alla prima traduzione integrale cui intelligentemente attese Riccardo Sonzogno (1893 e 1894).

Caproni dà prova di grande serietà e consapevolezza quando afferma che "Baudelaire è pressoché intraducibile", preferendo per questo "alla presunzione d'un rifacimento in versi italiani ... la semplice traduzione in prosa". Il lavoro si rivela particolarmente aderente all'originale e illuminato da soluzioni di grande eleganza nella loro semplicità. Soltanto un po' stupisce che in «Au Lecteur» il verbo "mange" del v. 17 sia stato tradotto da Caproni con uno "smunge", quando manger, piaccia o non piaccia, ha come unico significato quello di mangiare (molti sono i traduttori che non se la sono sentita di accettare l'inevitabile traduzione di "mange", cioè "mangia", ingegnandosi in vari modi per girarci intorno). A proposito di «Harmonie du soir», sarebbe stato auspicabile che "les temps" del verso d'apertura non si fosse ridotto e banalizzato con "la stagione", quando non c'è dubbio che Baudelaire abbia voluto indicare con quel plurale qualcosa 
di ben più vasto, quasi di profetico, appunto "i tempi", da intendersi nella loro valenza di età o epoca storica. Il "Paris" del penultimo verso del "Crépuscule du matin» è stato naturalmente reso al femminile ("la cupa Parigi"), quando invece il senso del testo richiede imperativamente un maschile ("le sombre Paris"). S'intende che questi rilievi non inficiano per nulla un lavoro traduttorio che resta della più alta qualità $e$ correttezza, sicuramente fra i migliori in assoluto.

L'impianto critico del libro è tenuto saldamente in mano da Luca Pietromarchi, la cui Introduzione costituisce uno dei saggi più eleganti e ricchi di spunti che si possano leggere nel quadro della vulgata baudelairiana. Quanto al commento, lo stesso Pietromarchi ci avvisa che esso rispetta "il principio della assoluta autonomia del testo nei confronti sia dei dati biografici che della cronologia di composizione, azzerata dalla logica della disposizione, ammettendo le sole influenze intertestuali generate dalla struttura dinamica e drammatica del canzoniere, nonché dal gioco di rimandi tematici che essa produce" (p. 44). Ogni singolo commento è corredato da una bibliografia mirata con riferimenti puntuali; mentre conclude il volume una equilibrata bibliografia essenziale. 